\title{
Matrine exerts anti-breast cancer activity by mediating apoptosis and protective autophagy via the AKT/mTOR pathway in MCF-7 cells
}

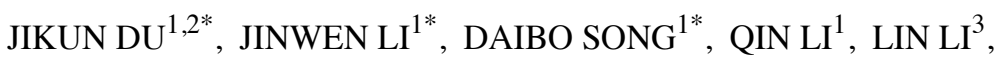 \\ BAOHONG LI ${ }^{1}$ and $\mathrm{LI} \mathrm{LI}^{1}$
}

\begin{abstract}
${ }^{1}$ Dongguan Scientific Research Center, Department of Pharmacology, Guangdong Medical University, Dongguan, Guangdong 523808; ${ }^{2}$ Central Research Laboratory, Shenzhen Hospital of Integrated Traditional Chinese and Western Medicine, The Second People's Hospital of Bao'an Shenzhen (Group), Shajing People's Hospital of Bao'an Shenzhen, Shenzhen, Guangdong 518104; ${ }^{3}$ Guangdong Provincial Key Laboratory of New Drug Screening, School of Pharmaceutical Sciences, Southern Medical University, Guangzhou, Guangdong 510515, P.R. China
\end{abstract}

Received March 1, 2020; Accepted June 11, 2020

DOI: $10.3892 / \mathrm{mmr} .2020 .11449$

\begin{abstract}
Matrine, a major alkaloid isolated from the traditional Chinese herb Sophora flavescens, has been used clinically to treat breast cancer in China. However, the effects of matrine on apoptosis and autophagy in breast cancer cells remain unclear. In the present study, the anti-breast cancer capacity of matrine was evaluated and its role in regulating apoptosis and autophagy in vitro was investigated. Matrine significantly inhibited the growth of MCF-7 cells. In addition, Hoechst 33342 staining and Annexin V/propidium iodide staining demonstrated that incubation with matrine induced apoptosis in MCF-7 cells. Furthermore, matrine induced autophagy in MCF-7 cells, manifesting as an accumulation of light chain 3 II and downregulation of p62. Additionally, matrine suppressed AKT and mammalian target of rapamycin (mTOR) phosphorylation, indicating that the AKT/mTOR pathway is involved in matrine-induced apoptosis and autophagy. Overall, the results of the present study indicated that matrine possesses anti-breast cancer activity by providing protective autophagy via inhibition of the AKT/mTOR pathway. These findings indicated that matrine may be a promising candidate for drug development targeting breast cancer.
\end{abstract}

Correspondence to: Professor $\mathrm{Li} \mathrm{Li}$, Dongguan Scientific Research Center, Department of Pharmacology, Guangdong Medical University, Xincheng Street, Songsan Lake, Dongguan, Guangdong 523808, P.R. China

E-mail: china_lovelylily@hotmail.com

*Contributed equally

Key words: matrine, MCF-7 cells, apoptosis, autophagy, breast cancer

\section{Introduction}

Breast cancer is a malignant disease that seriously damages the health of women worldwide and is considered to be the second leading cause of cancer mortality in women according to the 2018 American Cancer Report (1). Patients with breast cancer undergo surgery or radiation therapy combined with chemotherapy; despite the improved survival rates with these treatments, drug resistance and recurrence in certain patients continues to lead to poor prognosis and treatment failure (2). Therefore, developing effective adjuvant therapy with fewer side-effects compared with main treatments to reduce the progression of breast cancer has become an urgent need.

Natural products have been attracting increasing interest worldwide due to their extensive biological activities and minimum toxicity $(3,4)$. These natural products have contributed to improving the quality of life and several of them have been used clinically to treat various diseases, including cancer (5). Matrine is a bioactive compound found in the traditional Chinese medicine Sophora flavescens (Fig. 1). It demonstrates versatile pharmacological properties, including anti-inflammatory (6), antiviral (7), antitumor (8) and anti-fibrotic properties (9). Matrine has been demonstrated to effectively influence the development and progression of cancer involving the blockade of cell cycle progression (10) and the induction of apoptosis (11). Previous studies also confirmed that matrine suppressed proliferation, invasion and metastasis, regulating the expression of oncogenes and inhibiting the production of cytokines of breast cancer in vitro and in vivo $(12,13)$. Due to such promising efficacy, matrine is currently used as an adjuvant in China to improve 5-year survival rates and quality of life in breast cancer patients and to enhance their immunity (6). Nevertheless, the functional role and exact mechanisms of matrine against breast cancer remain to be elucidated.

Autophagy is an evolutionarily conserved mechanism responsible for cellular homeostasis in eukaryotic cells. It is a 
form of programmed cell death that regulates tumor development and progression $(14,15)$. In addition, autophagy can have opposing roles in different cell types (16), for example activation of autophagy can contribute to the survival of human lung carcinoma A549 cells (17), or promote cell death of U251 (14) and SMMC7721 cells (15). Autophagy triggered in response to anticancer therapy can be associated with cell death, leading to the activation of the pro-death signaling pathway in cancer cells (18). Previous studies demonstrated that autophagy is involved in breast cancer biology $(19,20)$ and in the development of therapy resistance in breast cancer (21). Recently, studies demonstrated that autophagy served a crucial role in breast cancer therapeutics. For example, baicalein was revealed to suppress cell proliferation and induce apoptosis and autophagy in breast cancer cells by inhibiting the PI3K/AKT pathway both in vitro and in vivo (22). In another study, corilagin was revealed to inhibit breast cancer growth via reactive oxygen species (ROS)-dependent apoptosis and autophagy (23). Studies have demonstrated that matrine possesses potential antitumor activities in breast cancer cells $(5,24)$. However, the exact effects of autophagy in regulating the cellular sensitivity of breast cancer to matrine treatment remain to be elucidated.

Therefore, the present study investigated the influence of matrine on breast cancer and explored the potential mechanisms of autophagy involved in the anti-breast cancer activity of matrine. To this end, a widely used epithelial cancer cell line MCF-7, derived from breast adenocarcinoma, was employed as an in vitro model to investigate the anticancer effects of matrine on breast cancer by focusing on its influence on autophagy. The findings of the present study may afford new insights into the mechanisms of matrine in inhibiting breast cancer.

\section{Materials and methods}

Materials. Matrine was purchased from Shanghai Yuanye Biotechnology Co.,Ltd.,(purity 98\%).DMEM was obtained from Gibco (Thermo Fisher Scientific, Inc.). Penicillin-streptomycin was purchased from Beijing Solarbio Science and Technology Co., Ltd. Fetal bovine serum (FBS) was purchased from Zhejiang Tianhang Biotechnology Co., Ltd. BCA protein assay kit and Annexin V-FITC apoptosis detection kit were provided by Beyotime Institute of Biotechnology. Cell Counting Kit- 8 (CCK-8) was obtained from Dojindo Molecular Technologies, Inc. Antibodies to light chain 3 (LC3)-II rabbit $\mathrm{mAb}$ (cat. no. 3868s), p62 rabbit mAb (cat. no. 5114), phosphorylated (p-)AKT rabbit mAb (cat. no. 13038), p-mammalian target of rapamycin (mTOR) rabbit $\mathrm{mAb}$ (cat. no. 5536), $\beta$-actin rabbit $\mathrm{mAb}$ (cat. no. 8457) and anti-rabbit IgG alkaline phosphatase (AP)-linked antibodies (cat. no. 7054) were obtained from Cell Signaling Technology, Inc.

Cell culture. MCF-7 cells were provided by Professor Jianqiang Liu (Guangdong Medical University, China). The cells were maintained in DMEM supplemented with 10\% FBS and 1\% penicillin-streptomycin at $37^{\circ} \mathrm{C}$ in a humidified atmosphere of $5 \% \mathrm{CO}_{2}$.

Cell viability assay. MCF-7 cells were seeded into the 96-well plate at $5 \times 10^{4}$ cells $/ \mathrm{ml}$. After the cells were attached, they were incubated with 2, 4, 8 and $10 \mathrm{mM}$ matrine for 24,48 and $72 \mathrm{~h}$. Cell viability of MCF-7 was determined using the CCK-8 assay kit in accordance with the manufacturer's protocol. The absorbance at $450 \mathrm{~nm}$ was measured with a microplate reader.

Morphological changes of MCF-7 cells. MCF-7 cells $\left(1 \times 10^{5}\right.$ cells $\left./ \mathrm{ml}\right)$ were seeded into 12 -well plates and the morphological changes were observed by optical microscope at X200 magnification and images were captured after the cells had been treated with matrine for $24 \mathrm{~h}$.

Apoptosis analysis. Following matrine exposure, MCF-7 were collected by scraping with a rubber policeman on the ice followed by centrifugation at $1,000 \mathrm{x} \mathrm{g}$ for $5 \mathrm{~min}$ at $4^{\circ} \mathrm{C}$ and re-suspended in fresh medium at a density of $2 \times 10^{5}$ cells $/ \mathrm{ml}$. Apoptosis was assessed according to the manufacturer's instructions. The cells were labeled with $5 \mu \mathrm{l}$ Annexin V-FITC and $5 \mu \mathrm{l}$ of propidium iodide (PI) for $10 \mathrm{~min}$ in the dark. Samples were analyzed using flow cytometry (FACSVerse; BD Biosciences) and data were analyzed with FlowJo 7.6.1 software (Tree star, Inc.). Annexin $\mathrm{V}^{+} / \mathrm{PI}^{-}$cells were identified as early apoptotic cells, whereas Annexin $\mathrm{V}^{+} / \mathrm{PI}^{+}$cells were designated as late apoptotic cells.

Hoechst 33342 staining. Apoptotic condensed nuclear changes were identified with Hoechst 33342 staining. The MCF-7 cells were washed twice with PBS and then incubated in Hoechst 33342 at $37^{\circ} \mathrm{C}$ for $30 \mathrm{~min}$ following matrine treatment. Then, alterations in nuclear morphology were imaged under a Olympus IX73 fluorescence microscope at $377 \mathrm{~nm}$ excitation and $447 \mathrm{~nm}$ emission wavelengths (Olympus Corporation) from five random fields (magnification, x200).

Western blot analysis. Total protein was extracted using RIPA buffer containing $1 \mathrm{mM}$ phenylmethylsulphonyl fluoride (Beyotime Institute of Biotechnology) at $4^{\circ} \mathrm{C}$ for $30 \mathrm{~min}$. The concentrations of the protein were determined using a BCA protein assay kit. Equal amounts of proteins $(20 \mu \mathrm{g})$ were subjected to $10 \%$ SDS-polyacrylamide gel electrophoresis and electro-transferred to Immuno-Blot PVDF membranes. Membranes were blocked with $5 \%(\mathrm{w} / \mathrm{v})$ bovine serum albumin (Beyotime Institute of Biotechnology, China) for $2 \mathrm{~h}$ at $4^{\circ} \mathrm{C}$. Subsequently, the membranes were incubated with primary antibody (LC3-II, p62, p-AKT, p-mTOR, and $\beta$-actin; all $1: 1,000)$ overnight at $4^{\circ} \mathrm{C}$. After washing with $0.1 \%(\mathrm{v} / \mathrm{v})$ Tween-20 in TBS, the anti-rabbit IgG AP-linked secondary antibody was applied for $1 \mathrm{~h}$ at room temperature. The bands were developed using the ECL detection system (Bio-Rad Laboratories, Inc.) and densitometry analysis was performed by ImageJ software (version 1.46, National Institutes of Health). $\beta$-actin served as the internal reference concurrently. All data were expressed as the relative intensity compared to the control group for the statistical analyses.

Statistical analysis. Data were reported as the means \pm standard deviation of at least three independent experiments conducted in triplicate. Statistical analyses were performed using GraphPad Prism 8.0 software (GraphPad Software, Inc.). Differences between groups were performed using Student's t-test (2-group comparisons) or one-way analysis of 

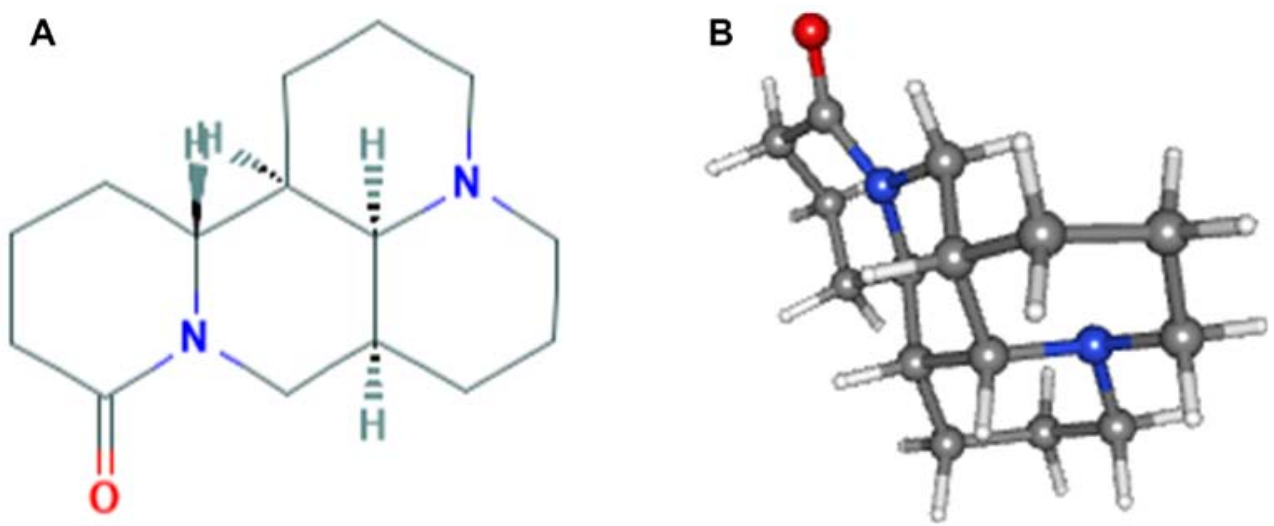

Figure 1. Chemical structure of matrine. (A) 2-D Structure of matrine and (B) 3-D conformer of matrine. Chemical formula: $\mathrm{C}_{15} \mathrm{H}_{24} \mathrm{~N}_{2} \mathrm{O}$, molecular weight: $248.37 \mathrm{~g} / \mathrm{mol}$, from PubChem https://pubchem.ncbi.nlm.nih.gov/compound/91466.

variance followed by Tukey's post hoc test ( $>2$ groups). $\mathrm{P}<0.05$ was considered to indicate a statistically significant difference.

\section{Results}

Matrine decreases cell viability of MCF-7 cells. To elucidate the impact of matrine on the cell viability of human breast cancer MCF-7 cells, the cells were cultured with 2, 4, 8 and $10 \mathrm{mM}$ matrine for 24,48 and $72 \mathrm{~h}$, following which the CCK-8 assay was conducted. As revealed in Fig. 2, matrine significantly caused the inhibition of the viability of MCF-7 cells $(\mathrm{P}<0.05)$ and this effect was dose-dependent. Over the course of extended treatments $(72 \mathrm{~h})$, matrine exhibited a greater inhibitory effect compared with that at 24 and $48 \mathrm{~h}$ $(\mathrm{P}<0.01)$. These results indicated that matrine exhibited anti-cell growth effects on breast cancer MCF-7 cells in a dose- and time-dependent manner.

Matrine induces morphologic changes of MCF-7 cells. Morphological features were observed in MCF-7 cells following treatment with various concentrations of matrine. MCF-7 cells demonstrated shrinkage, membrane blebbing, ballooning and partial detachment after treatment with matrine for $24 \mathrm{~h}$ (Fig. 3).

Matrine induces cell apoptosis in MCF-7 cells. To evaluate the effect of matrine on the apoptosis of breast cancer MCF-7 cells, Hoechst 33342 staining, which is sensitive to DNA, was used to estimate the changes of nuclear morphology. As revealed in Fig. 4A, Hoechst 33342 staining of the control MCF-7 cells exhibited a uniformly blue dyed pattern in the nuclei whereas the cells incubated with matrine demonstrated typical characteristics of apoptosis, namely nuclear condensation/fragmented chromatin and apoptotic bodies with bright blue fluorescent dots. These data indicated that matrine could effectively induce cellular apoptosis. Apoptotic responses were also assessed by flow cytometry following Annexin V/PI staining. As is revealed in Fig. 4B, exposure to matrine led to a significant increase in the percentage of apoptotic cells in MCF-7 cells compared with the control. The apoptotic rates were $56.04 \pm 2.00 \%(2 \mathrm{mM}), 64.28 \pm 2.68 \%(4 \mathrm{mM})$ and $72.81 \pm 3.83 \%(8 \mathrm{mM})$, respectively. These results further confirmed that matrine could induce cellular apoptosis. Based

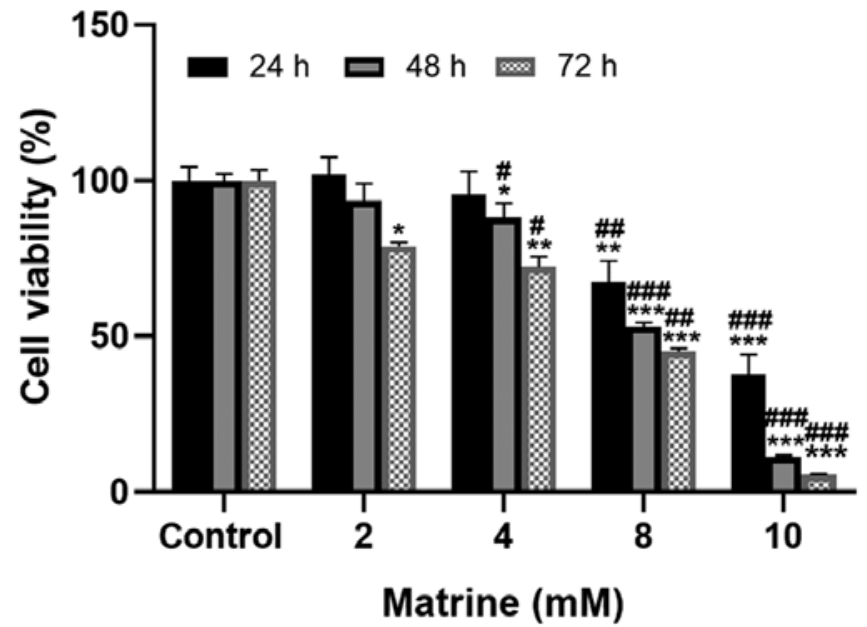

Figure 2. Effects of matrine on the cell viability of human breast cancer MCF-7 cells. MCF-7 cells were treated with 2, 4, 8 and $10 \mathrm{mM}$ matrine for 24,48 and $72 \mathrm{~h}$. Subsequently, cell viability was measured using a CCK-8 assay. The data are expressed as the means \pm standard deviation from three independent experiments. " $\mathrm{P}<0.05$ vs. the control group; ${ }^{* *} \mathrm{P}<0.01$ vs. the control group; ${ }^{* * *} \mathrm{P}<0.001$ vs. the control group; ${ }^{\text {" }} \mathrm{P}<0.05$ vs. $2 \mathrm{mM}$ groups;

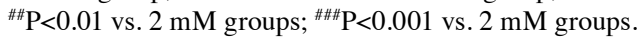

on these findings, matrine could induce an apoptotic response in MCF-7 cells.

Matrine induces autophagy in MCF-7 cells. To determine the effect of matrine on the induction of autophagy, expression of LC3-II, a protein recruited to autophagosomal membranes as an indication of autophagy (25), was assessed using western blotting. As demonstrated in Fig. 5, in MCF-7 cells, matrine enhanced LC3-II expression compared with the control group, indicating that matrine induced autophagic flux. To further determine whether matrine modulates autophagic degradation, the expression levels of p62, also known as sequestosome-1, which is associated with LC3-II turnover and functions as a marker of autophagic degradation (26) were assessed. MCF-7 cells incubated with matrine resulted in significantly decreased expression levels of p62, indicating an inductive effect of matrine on autophagic flux. Collectively, these results indicated that matrine was an inducer of autophagy. 

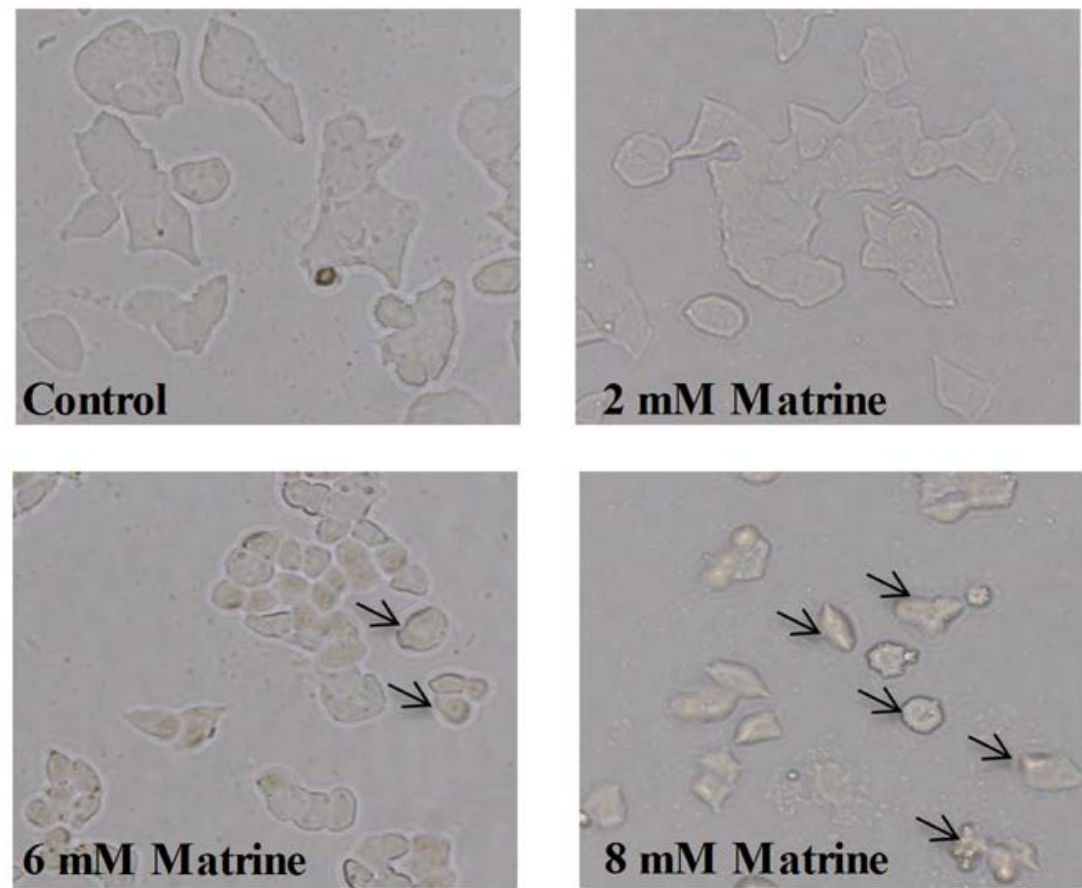

Figure 3. Effects of matrine on morphologic changes of human breast cancer MCF-7 cells. MCF-7 cells were treated with 2, 4 and $8 \mathrm{mM}$ matrine for $24 \mathrm{~h}$. The morphological features were observed using microscopy at x200 magnification. MCF-7 cells incubated with matrine demonstrated shrinkage, membrane blebbing, ballooning and partial detachment.
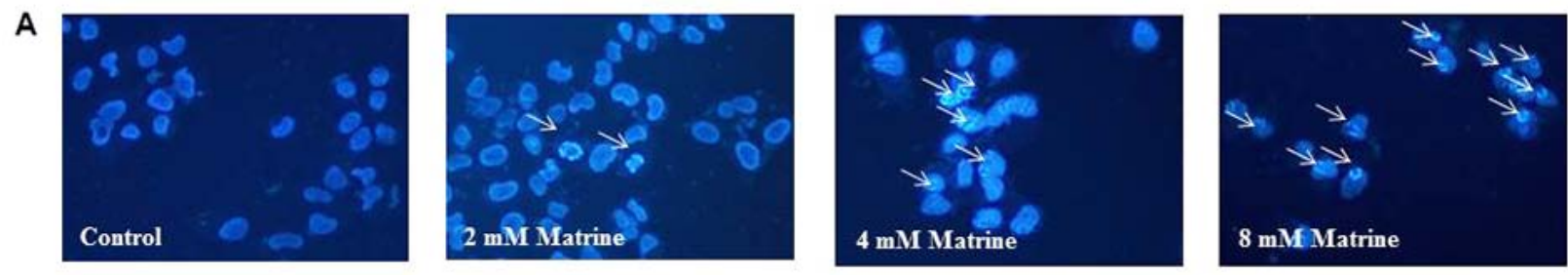

B
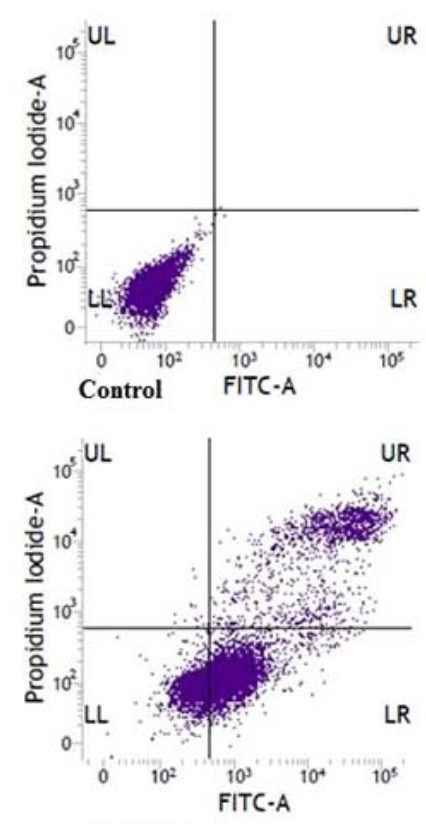

$4 \mathrm{mM}$ Matrine
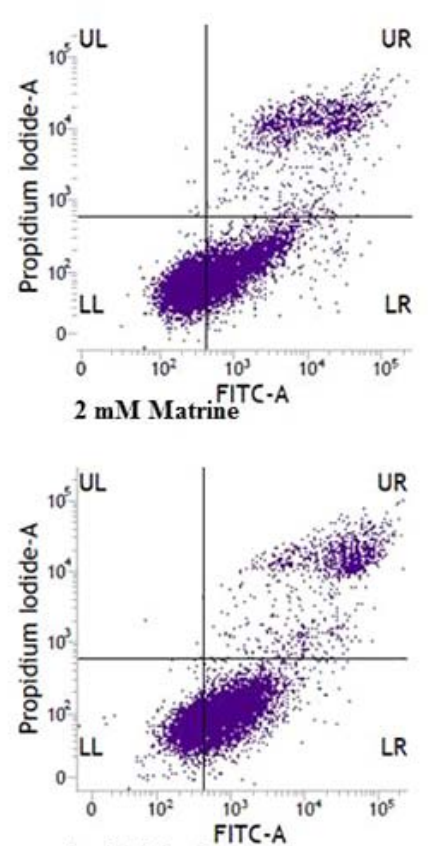

$8 \mathrm{mM}$ Matrine

Figure 4. Effects of matrine on human breast cancer MCF-7 cell apoptosis. MCF-7 cells were treated with 2, 4 and 8 mM matrine for 24 h. (A) Apoptotic condensed nuclear changes were quantified using Hoechst 33342 staining and observed using fluorescence microscopy at x200 magnification. (B) Apoptosis was determined by Annexin V-FITC/PI staining and flow cytometry. Statistical analysis was performed using one-way ANOVA followed by Tukey's multiple comparison test; ${ }^{* * *} \mathrm{P}<0.001$ vs. the control group; ${ }^{\#} \mathrm{P}<0.05$ and ${ }^{\# \# \#} \mathrm{P}<0.001$ vs. the $2 \mathrm{mM}$ matrine group. UL: Upper left, UR: Upper right, LL: Lower left, LR: Lower right. 

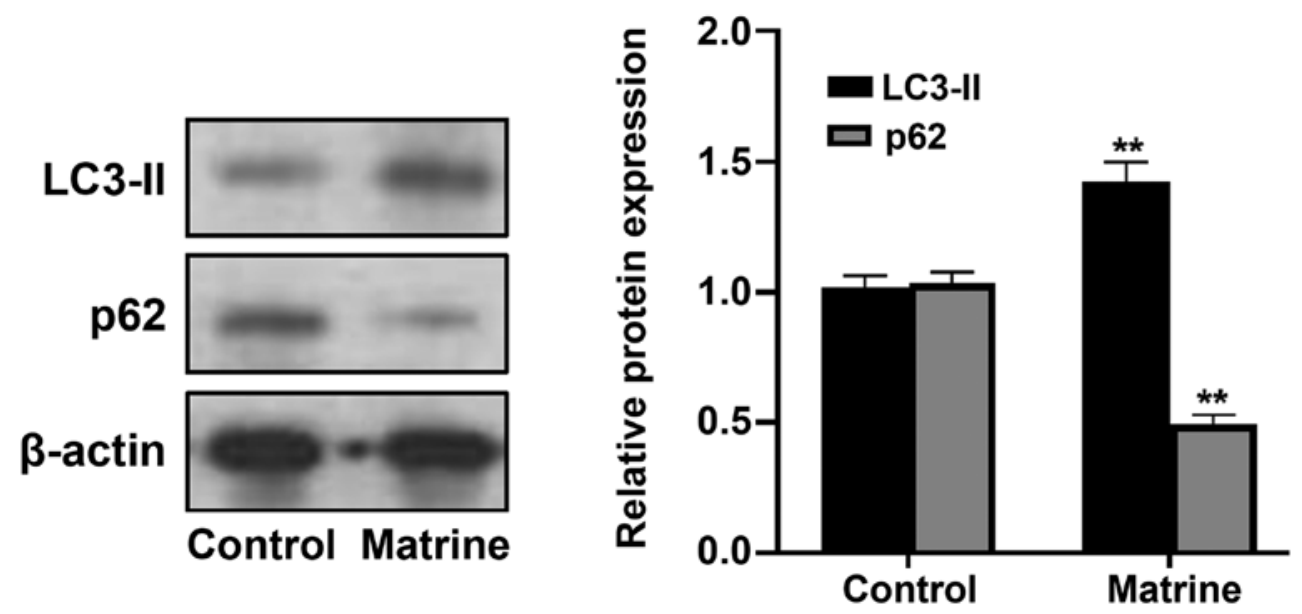

Figure 5. Effects of matrine in inducing autophagy in breast cancer MCF-7 cells. MCF-7 cells were treated with and without matrine for $24 \mathrm{~h}$. The levels of the autophagy-related protein p 62 and LC3-II were confirmed using western blotting, with $\beta$-actin used as loading control. LC 3 , light chain 3 . ${ }^{* *} \mathrm{P}<0.01$ vs. the control group.
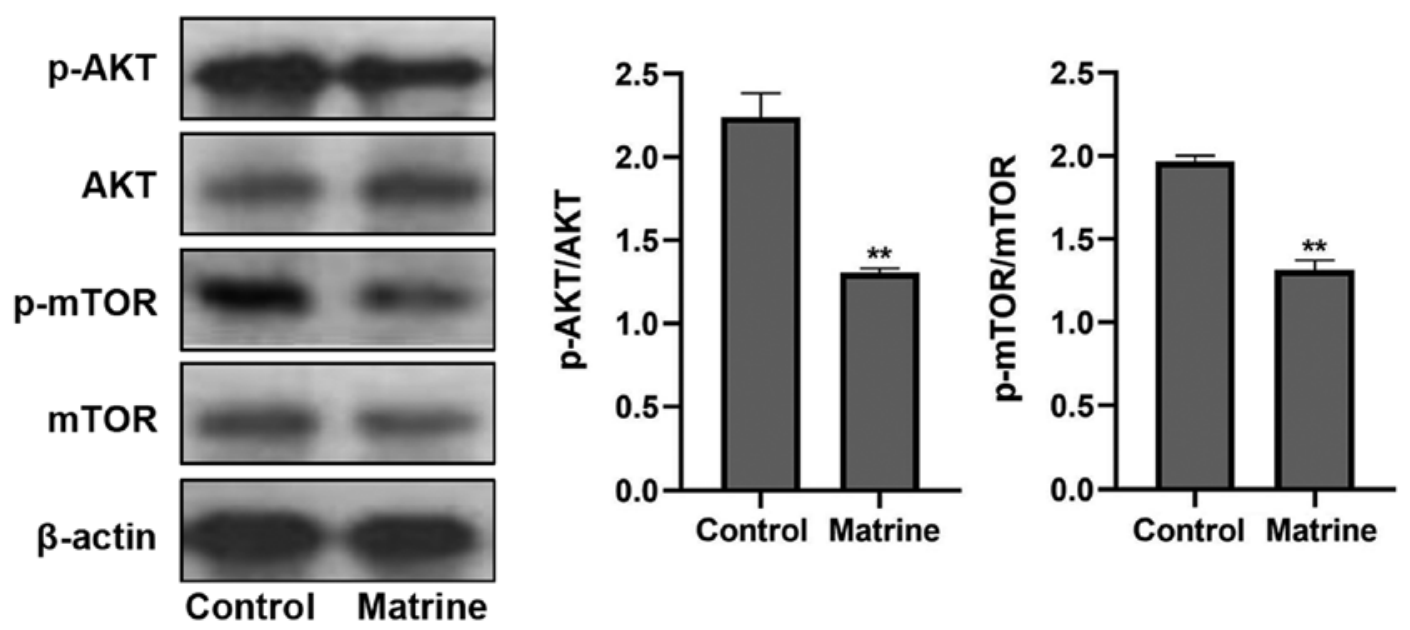

Figure 6. Effects of matrine on the activation of the AKT/mTOR pathway. MCF-7 cells were treated with and without matrine for $24 \mathrm{~h}$. Cell extracts were analyzed by western blotting with antibodies specific for p-AKT and p-mTOR. Quantification of protein expression levels of p-AKT and p-mTOR to $\beta$-actin. ${ }^{* *} \mathrm{P}<0.01$ vs. the control group. $\mathrm{p}-$, phosphorylated; mTOR, mammalian target of rapamycin.

Matrine induces autophagy via the AKT/mTOR pathway. Next, the pathways involving the activation of autophagy induced by matrine analysis were explored using western blotting. It was identified that, compared with the control group, levels of phosphorylation of AKT and mTOR were significantly downregulated in MCF-7 cells following incubation with matrine (Fig. 6). Matrine treatment significantly decreased the ratio of p-AKT/AKT in MCF-7 cells. Variation in the $\mathrm{p}-\mathrm{mTOR} / \mathrm{mTOR}$ ratio was consistent with the change in the p-AKT/AKT ratio. Collectively, these results indicated that matrine-induced autophagy was due, at least in part, to inhibition of the AKT/mTOR pathway.

\section{Discussion}

Breast cancer is the commonest type of malignancy in females worldwide (27). Recently, phytochemicals have become a substantial source of cancer treatments (28). Matrine, an alkaloid component extracted from the Chinese herb Sophora flavescens, is considered to improve the quality of life and prognosis of cancer patients when combined with standard therapies (29). Matrine exhibits pro-apoptotic (30), anti-proliferation (31) and metastasis-suppression effects $(32,33)$ in various cancer cells such as SGC-7901 human gastric cancer cell and osteosarcoma cells $(12,13)$. However, the mechanisms underlying the anticancer properties of matrine in MCF-7 breast cancer cells remain to be elucidated. Therefore, the potential mechanism of matrine against MCF-7 breast cancer cells was explored in the present study.

To determine the role of matrine in MCF-7 breast cancer cells, CCK-8 assay was performed. Consistent with previous studies $(6,34)$, matrine significantly suppressed cell viability in a dose- and time-dependent manner in MCF-7 breast cancer cells. Furthermore, the morphological changes of the MCF-7 breast cancer cells cultured in the presence of matrine also revealed its cytotoxic effect on MCF-7 cells. Apoptosis induction serves a key role in cancer therapy (35). In the present study, Hoechst 33342 staining and flow cytometry revealed that matrine could cause cellular apoptosis in MCF-7 cells. These findings supported the hypothesis that inhibition of 
cellular growth in MCF-7 by matrine was at least partly attributed to its induction of apoptosis.

Autophagy is closely associated with cancer therapeutics (36). Previous studies have revealed that autophagy enhancers can be used as potential treatment strategies for cancer in precancerous lesions $(37,38)$. In advanced cancer, intervening in autophagy has been suggested as one of the effective measures for cancer treatment $(39,40)$. Resveratrol has been revealed to inhibit the proliferation of A549 lung cancer cells and to promote cell death by an autophagic mechanism (41). Juglone conferred protection in human hepatocellular carcinoma cells, mediated by its ability to induce apoptosis and autophagy by adjustment of mitogen-activated protein kinase pathways (42). Recently, several lines of evidence have also demonstrated that matrine has potential anticancer properties by enhancing autophagy. Matrine can induce apoptosis and autophagy of glioma cell line U251 via modulation of circular RNA-104075/BCL-9 (43) and induced protective autophagy via ERK activation in osteosarcoma cells (30). However, there is insufficient evidence to determine the role of matrine interaction between autophagy and breast cancer. To the best of the authors' knowledge, the present study is the first time to identify matrine as an inducer of autophagy in breast cancer MCF-7 cells. LC3, a cytosolic ubiquitin-like protein, is expressed in the majority of cell types (44). During autophagosome formation, cytosolic LC3-I is transformed into its enzymatic LC3-II counterpart that aggregates on membranes of autophagosomes (45); p62, a ubiquitin-binding scaffold protein, has an LC3-interacting domain and is degraded during autophagy progression (46). LC3-II and p62 are therefore reliable autophagy markers. Thus, to determine the role of matrine in autophagy induction, expression levels of LC3-II and p62 were assessed using western blotting. The results demonstrated that matrine upregulated the expression of LC3-II and concomitantly decreased p62 expression. These results indicated that the cytotoxic effects of matrine in breast cancer MCF-7 cells may at least in part associated with the induction of autophagy.

Autophagy is regulated by a number of upstream signaling pathways (47). The AKT/mTOR pathway is a crucial signaling cascade that regulates numerous cellular processes in both normal and cancer cells, including cell proliferation, the cell cycle, synthesis of protein and angiogenesis (48). mTOR is phosphorylated by activated AKT and p-mTOR negatively regulates autophagy (49). A previous study suggested that the AKT/mTOR signaling pathway serves a vital role in autophagy (50). Inhibition of the AKT/mTOR pathway was revealed to promote autophagy and apoptosis to produce anticancer effects (51). Triptolide suppressed tumor growth by inducing apoptosis and autophagy by activating the ROS/JNK and blocking the AKT/mTOR signaling pathways in glioma cells (52). Curcumin demonstrated its antitumor activity by regulating autophagy in human lung cancer A549 cells by inhibiting the PI3K/AKT/mTOR pathway (53). The present study determined whether the AKT/mTOR signaling pathway could be responsible for the autophagy upregulation efficacy of matrine. Matrine treatment significantly inhibited the phosphorylation of AKT and mTOR in breast cancer MCF-7 cells. Collectively, the data from the present study indicated that the AKT/mTOR signaling pathway participated in matrine-induced apoptosis and protective autophagy in breast cancer MCF-7 cells. The present study demonstrated that, in breast cancer MCF-7 cells, matrine could suppress cellular growth by triggering apoptosis and autophagy, both of which are regulated by the AKT/mTOR signaling pathway. Consequently, these findings indicated that matrine has a potential application in the treatment of human breast cancer. However, the several factors involved in these events are complex processes that require further characterization. Detailed in vivo investigations into the therapeutic benefits of matrine should be pursued.

\section{Acknowledgements}

Not applicable.

\section{Funding}

The present study was supported by the Guangdong Natural Science Foundation (grant nos. 2018A030313084 and 2018A030310112), the Administration of Traditional Chinese Medicine of Guangdong Province of China (grant nos. 20181150 and 20191187), the 'Group-type' Special Support Project for Education Talents in Universities (grant no. 4SG19045G), Public Service Platform Open Project Fund of South China Sea for R\&D Marine Biomedicine Resources (grant no. 2HC18010) and the Shenzhen Bao'an Science and Technique Project (grant no. 2019JD214).

\section{Availability of data and materials}

The datasets used and/or analyzed during the current study are available from the corresponding author on reasonable request.

\section{Authors' contributions}

JD, LinL and LiL conceived the study. BL and LiL designed the study. JL, DS, QL and LiL performed the experiments. JL and LiL analyzed the data. JD and LiL wrote the manuscript. All authors read and approved the final manuscript.

\section{Ethics approval and consent to participate}

Not applicable.

\section{Patient consent for publication}

Not applicable.

\section{Competing interests}

The authors declare that they have no competing interests.

\section{References}

1. Siegel RL, Miller KD and Jemal A: Cancer statistics, 2018. CA Cancer J Clin 60: 277-300, 2018.

2. Yang LH, Ho YJ, Lin JF, Yeh CW, Kao SH and Hsu LS: Butein inhibits the proliferation of breast cancer cells through generation of reactive oxygen species and modulation of ERK and p38 activities. Mol Med Rep 6: 1126-1132, 2012. 
3. Du J, Li L and Zhou S: Microbial production of cyanophycin: From enzymes to biopolymers. Biotechnol Adv 37: 107400, 2019

4. Du J, Yang D, Luo ZW and Lee SY: Metabolic engineering of Escherichia coli for the production of indirubin from glucose. J Biotechnol 267: 19-28, 2018.

5. Brower V: Back to nature: Extinction of medicianl plants threathens drug discovery. J Natl Cancer Inst 100: 838-839, 2008.

6. Zhou BG, Wei CS, Zhang S, Zhang Z and Gao HM: Matrine reversed multidrug resistance of breast cancer MCF-7/ADR cells through PI3K/AKT signaling pathway. J Cell Biochem 119 3885-3891, 2018

7. Zhang YB, Zhan LQ, Li GQ, Wang F, Wang Y, Li YL, Ye WC and Wang GC: Dimeric matrine-type alkaloids from the roots of Sophora flavescens and their anti-hepatitis B virus activities. J Org Chem 81: 6273-6280, 2016.

8. Zhang Y, Zhang H, Yu P, Liu Q, Liu K, Duan H, Luan G, Yagasaki $\mathrm{K}$ and Zhang G: Effects of matrine against the growth of human lung cancer and hepatoma cells as well as lung cancer cell migration. Cytotechnology 59: 191-200, 2009.

9. Zhang Y, Cui L, Guan G, Wang J, Qiu C, Yang T, Guo Y and Liu Z: Matrine suppresses cardiac fibrosis by inhibiting the TGF- $\beta$ /Smad pathway in experimental diabetic cardiomyopathy. Mol Med Rep 17: 1775-1781, 2018

10. Zhang P, Wang Z, Chong T and Ji Z: Matrine inhibits proliferation and induces apoptosis of the androgen-independent prostate cancer cell line PC-3. Mol Med Rep 5: 783-787, 2012

11. Zhou N, Li J, Li T, Chen G, Zhang Z and Si Z: Matrine-induced apoptosis in Hep3B cells via the inhibition of MDM2. Mol Med Rep 15: 442-450, 2016.

12. Huang $\mathrm{J}$ and $\mathrm{Xu} \mathrm{H}$ : Matrine: Bioactivities and structural modifications. Curr Top Med Chem 16: 3365-3378, 2016.

13. Yong J, Wu X and $\mathrm{Lu} \mathrm{C}$ : Anticancer advances of matrine and its derivatives. Curr Pharm Des 21: 3673-3680, 2015.

14. Xue S, Zhou Y, Zhang J, Xiang Z, Liu Y, Miao T, Liu G, Liu B, Liu X, Shen L, et al: Anemoside B4 exerts anti-cancer effect by inducing apoptosis and autophagy through inhibiton of $\mathrm{PI} 3 \mathrm{~K} / \mathrm{Akt} / \mathrm{mTOR}$ pathway in hepatocellular carcinoma. Am J Transl Res 11: 2580-2589, 2019.

15. Guo Z, Guozhang H, Wang H, Li Z and Liu N: Ampelopsin inhibits human glioma through inducing apoptosis and autophagy dependent on ROS generation and JNK pathway. Biomed Pharmacother 116: 108524, 2019.

16. Liu J, Fan L, Wang H and Sun G: Autophagy, a double-edged sword in anti-angiogenesis therapy. Med Oncol 33: 10, 2016.

17. Kaminskyy VO, Piskunova T, Zborovskaya IB, Tchevkina EM and Zhivotovsky B: Suppression ofbasal autophagy reduces lung cancer cell proliferation and enhancescaspase-dependent and -independent apoptosis by stimulating ROS formation. Autophagy 8: 1032-1044, 2012.

18. Leidal AM, Levine B and Debnath J: Autophage and the cell biology of age-related disease. Nat Cell Biol 20: 1338-1348, 2018

19. Maycotte P, Gearheart CM,Barnard R,Aryal S, Mulcahy Levy JM, Fosmire SP, Hansen RJ, Morgan MJ, Porter CC, Gustafson DL and Thorburn A: STAT3-mediated autophagy dependence identifies subtypes of breast cancer where autophagy inhibition can be efficacious. Cancer Res 74: 2579-2590, 2014.

20. Lefort S, Joffre C, Kieffer Y, Givel AM, Bourachot B, Zago G, Bieche I, Dubois T, Meseure D, Vincent-Salomon A, et al: Inhibition of autophagy as a new means of improving chemotherapy efficiency in high-LC3B triple-negative breast cancers. Autophagy 10: 2122-2142, 2014.

21. Cook KL, Wärri A, Soto-Pantoja DR, Clarke PA, Cruz MI, Zwart A and Clarke R: Hydroxychloroquine inhibits autophagy to potentiate antiestrogen responsiveness in ER+ breast cancer Clin Cancer Res 20: 3222-3232, 2014.

22. Yan W, Ma X, Zhao X and Zhang S: Baicalein induces apoptosis and autophagy of breast cancer cells via inhibiting PI3K/AKT pathway in vivo and vitro. Drug Des Devel Ther 12: 3961-3972, 2018.

23. Tong Y, Zhang G, Li Y, Xu J, Yuan J, Zhang B, Hu T and Song G: Corilagin inhibits breast cancer growth via reactive oxygen species-dependent apoptosis and autophagy. J Cell Mol Med 22: 3795-3807, 2018.

24. Xiao X, Ao M, Xu F, Li X, Hu J, Wang Y, Li D, Zhu X, Xin C and Shi W: Effect of matrine against breast cancer by downregulating the vascular endothelial growth factor via the $\mathrm{Wnt} / \beta$-catenin pathway. Oncol Lett 15: 1691-1697, 2018.
25. Xie Z, Xie Y, Xu Y, Zhou H, Xu W and Dong Q: Bafilomycin A1 inhibits autophagy and induces apoptosis in MG63 osteosarcoma cells. Mol Med Rep 10: 1103-1107, 2014.

26. Yue Z: Regulation of neuronal autophagy in axon: Implication of autophagy in axonal function and dysfunction/degeneration. Autophagy 3: 139-141, 2007.

27. Jemal A, Bray F, Center MM, Ferlay J, Ward E and Forman D: Global cancerstatistics. CA Cancer J Clin 61: 69-90, 2011.

28. Efferth T, Konkimalla VB, Wang YF, Sauerbrey A, Meinhardt S, Zintl F, Mattern J and Volm M: Prediction of broad spectrum resistance of tumors towards anticancer drugs. Clin Cancer Res 14: 2405-2412, 2008.

29. Niu H, Zhang Y, Wu B, Zhang Y, Jiang H and He P: Matrine induces the apoptosis of lung cancer cells through downregulation of inhibitor of apoptosis proteins and the Akt signaling pathway. Oncol Rep 32: 1087-1093, 2014.

30. Ma K, Huang MY, Guo YX and Hu GQ: Matrine-induced autophagy counteracts cell apoptosis via the ERK signaling pathway in osteosarcoma cells. Oncol Lett 12: 1854-1860, 2016.

31. Yang W, Hosford SR, Traphagen NA, Shee K, Demidenko E, Liu S and Miller TW: Autophagy promotes escape from phosphatidylinositol 3-kinase inhibition in estrogen receptor-positive breast cancer. FASEB J 32: 1222-1235, 2018.

32. Yu P, Liu Q, Liu K, Yagasaki K, Wu E and Zhang G: Matrine suppresses breast cancer cell proliferation and invasion via VEGF-Akt-NF-kappaB signaling. Cytotechnology 59: 219-229, 2009.

33. Li H, Tan G, Jiang X, Qiao H, Pan S, Jiang H, Kanwar JR and Sun X: Therapeutic effects of matrine on primary and metastatic breast cancer. Am J Chin Med 38: 1115-1130, 2010.

34. Li H, Li X, Bai M, Suo Y, Zhang G, Cao X: Matrine inhibited proliferation and increased apoptosis in human breast cancer MCF-7 cells via upregulation of Bax and downregulation of Bcl-2. Int J Clin Exp Pathol 8: 14793-14799, 2015.

35. Tsuji T, Ozasa H, Aoki W, Aburaya S, Yamamoto Funazo T, Furugaki K, Yoshimura Y, Yamazoe M, Ajimizu H Yasuda Y, et al: YAP1 mediates survival of ALK-rearranged lung cancer cells treated with alectinib via pro-apoptotic protein regulation. Nat Commun 11: 74, 2020.

36. Wang S, Wang K, Wang H, Han J and Sun H: Autophagy is essential for flavopiridol-induced cytotoxicity against MCF-7 breast cancer cells. Mol Med Rep 16: 9715-9720, 2017.

37. Poillet-Perez L and White E: Role of tumor and host autophagy in cancer metabolism. Genes Dev 33: 610-619, 2019.

38. Chen J, Zhang L, Zhou H, Wang W, Luo Y, Yang H and Yi H: Inhibition of autophagy promotes cisplatin-induced apoptotic cell death through Atg5 and Beclin 1 in A549 human lung cancer cells. Mol Med Rep 17: 6859-6865, 2018.

39. Vidoni C,Ferraresi A, Secomandi E, Vallino L, Dhanasekaran DN and Isidoro C: Epigenetic targeting of autophagy for cancer prevention and treatment by natural compounds. Semin Cancer Biol, 2019 (Epub ahead of print).

40. Udristioiu A and Nica-Badea D: Autophagy dysfunctions associated with cancer cells and their therapeutic implications. Biomed Pharmacother 115: 108892, 2019.

41. Wang J,Li J, Cao N, LiZ, Han J and Li L: Resveratrol, an activator of SIRT1, induces protective autophagy in non-small-cell lung cancer via inhibiting Akt/mTOR and activating p38-MAPK. Onco Targets Ther 11: 7777-7786, 2018.

42. Wang P, Gao C, Wang W, Yao LP, Zhang J, Zhang SD, Li J, Fang $\mathrm{SH}$ and $\mathrm{Fu}$ YJ: Juglone induces apoptosis and autophagy via modulation of mitogen-activated protein kinase pathways in human hepatocellular carcinoma cells. Food Chem Toxicol 116: 40-50, 2018.

43. Chi G, Xu D, Zhang B and Yang F: Matrine induces apoptosis and autophagy of glioma cell line $\mathrm{U} 251$ by regulation of circRNA-104075/BCL-9. Chem Biol Interact 308: 198-205, 2019.

44. Saha S, Panigrahi DP, Patil S and Bhutia SK: Autophagy in health and disease: A comprehensive review. Biomed Pharmacother 104: 485-495, 2018.

45. Tazawa H, Kuroda S, Hasei J, Kagawa S and Fujiwara T: Impact of autophagy in oncolytic adenoviral therapy for cancer. Int J Mol Sci 18: 1479, 2017.

46. Cohen-Kaplan V, Livneh I, Avni N, Fabre B,Ziv T, Kwon YT and Ciechanover A: p62- and ubiquitin-dependent stress-induced autophagy of the mammalian $26 \mathrm{~S}$ proteasome. Proc Natl Acad Sci USA 113: E7490, 2016. 
47. Chen Y, Zhao X, Li J, Zhang L, Li R, Zhang H, Liao R, Liu S, Shi W and Liang X: Amino acid starvation promotes podocyte autophagy through mammalian target of rapamycin inhibition and transcription factor EB activation. Mol Med Rep 18: 4342-4348, 2018.

48. Heras-Sandoval D, Pérez-Rojas JM, Hernández-Damián J and Pedraza-Chaverri J: The role of PI3K/AKT/mTOR pathway in the modulation of autophagy and the clearance of protein aggregates in neurodegeneration. Cell Signal 26: 2694-2701, 2014.

49. Zhang XR, Wang SY, Sun W, Wei C: Isoliquiritigenin inhibits proliferation and metastasis of MKN28 gastric cancer cells by suppressing the PI3K/AKT/mTOR signaling pathway. Mol Med Rep 18: 3429-3436, 2018

50. Yu H, Qiu Y, Pang X, Li J, Wu S, Yin S, Han L, Zhang Y, Jin C, Gao X, et al: Lycorine promotes autophagy and apoptosis via TCRP1/Akt/mTOR axis inactivation in human hepatocellular carcinoma. Mol Cancer Ther 16: 2711-2723, 2017.
51. Hu JL, Hu XL, Guo AY, Wang CJ, Wen YY and Cang SD: Endoplasmic reticulum stress promotes autophagy and apoptosis and reverses chemoresistance in human ovarian cancer cells. Oncotarget 8: 49380-49394, 2017.

52. Liu X, Zhao P, Wang X, Wang L, Zhu Y and Gao W: Triptolide induces glioma cell autophagy and apoptosis via upregulating the ROS/JNK and downregulating the Akt/mTOR signaling pathways. Front Oncol 9: 387, 2019.

53. Liu F, Gao S, Yang Y, Zhao X, Fan Y, Ma W, Yang D, Yang A and Yu Y: Antitumor activity of curcumin by modulation of apoptosis and autophagy in human lung cancer A549 cells through inhibiting PI3K/Akt/mTOR pathway. Oncol Rep 39: 1523-1531, 2018.

This work is licensed under a Creative Commons

Attribution-NonCommercial-NoDerivatives 4.0 International (CC BY-NC-ND 4.0) License. 